\title{
2012 Journal of Global Optimization best paper award
}

\author{
Sergiy Butenko
}

Received: 30 January 2013 / Accepted: 4 February 2013 / Published online: 14 February 2013

(C) Springer Science+Business Media New York 2013

As mentioned in the previous editorials, thanks to the leadership of the previous Editorin-Chief, Panos Pardalos, and owing to the generous support of Springer, selected papers published in the Journal of Global Optimization (JOGO) will now be recognized by the Best Paper Award. The winners of this annual prize, which consists of $\$ 1,000$ and a certificate, are selected by an award committee consisting of the Editor-in-Chief and several invited members of the editorial board.

The inaugural 2012 Best Paper Award Committee included Panos Pardalos, Hanif Sherali, Ding-Zhu Du, Christodoulos Floudas, and Antanas Zilinskas, and considered papers published in the journal in 2011. I am pleased to announce that the winners of the Journal of Global Optimization Best Paper Award for 2011 are Joseph K. Scott, Matthew D. Stuber, and Paul I. Barton for their paper titled Generalized McCormick Relaxations. More detail is provided in the award citation below.

Congratulations to the winners, and thanks to Professor Pardalos and Vaishali Damle of Springer for establishing this important award, as well as to everyone involved in the committee and nomination process. I would also like to use this opportunity to invite nominations for the 2013 Best Paper Award. Any JOGO paper with a 2012 publication date (volumes 52-54) is eligible. The award committee is about to be formed; in the meantime, please submit your nominations to my electronic mail address (butenko@tamu.edu), and they will be forwarded to the committee. 
2012 Journal of Global Optimization Best Paper Award (for a paper published in 2011)

Authors: Joseph K. Scott, Matthew D. Stuber, and Paul I. Barton

Title: Generalized McCormick Relaxations

Published in: Volume 51, Number 4, pages 569-606 (2011)

Convex and concave relaxations are in the core of many global optimization algorithms. In 1976, McCormick proposed a recursive method to define convex underestimators for factorable functions, referred to as McCormick relaxations. Scott, Stuber, and Barton examine the McCormick relaxations from a new vantage point, unveiling new unexplored horizons for their applicability. Aiming to provide convex/concave relaxations for functions defined implicitly as the parametric solution of a system of equations, the authors make a major step towards achieving this ambitious goal by developing a method yielding relaxations of approximate solutions. Moreover, they design an algorithm for generating McCormick relaxations for the parametric solutions of ODEs, which are extendable to a wide range of optimal control problems. In addition, the groundwork for using these relaxations within a branchand-bound framework is laid by establishing finite convergence 